\title{
Differential regulation of $m s x$ genes in the development of the gonopodium, an intromittent organ, and of the "sword," a sexually selected trait of swordtail fishes (Xiphophorus)
}

\author{
Hans Zauner, ${ }^{a, 1,2}$ Gerrit Begemann, ${ }^{\text {a,1 }}$ Manuel Marí-Beffa, ${ }^{\mathrm{b}}$ and Axel Meyer ${ }^{\mathrm{a}, *}$

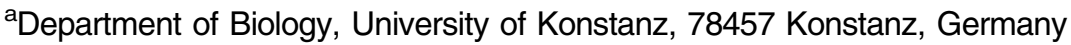 \\ bepartment of Cell Biology and Genetics, Faculty of Science, University of Málaga, Spain \\ *Author for correspondence (e-mail: axel.meyer@uni-konstanz.de) \\ ${ }^{1}$ Authors contributed equally to this work. \\ 2Present address: Max-Planck-Institute for Developmental Biology, Department IV, 72076 Tübingen, Germany.
}

\begin{abstract}
SUMMARY The possession of a conspicuous extension of colored ventral rays of the caudal fin in male fish of swordtails (genus Xiphophorus) is a prominent example for a trait that evolved by sexual selection. To understand the evolutionary history of this so-called sword molecularly, it is of interest to unravel the developmental pathways responsible for extended growth of sword rays during development of swordtail males. We isolated two msx genes and showed that they are differentially regulated during sword outgrowth. During sword growth in juvenile males, as well as during testosteroneinduced sword development and fin ray regeneration in the sword after amputation, expression of $m s x C$ is markedly upregulated in the sword forming fin rays. In contrast, $m s x E / 1$ is not differentially expressed in ventral and dorsal male fin rays,
\end{abstract}

suggesting a link between the development of male secondary sexual characters in fins and up-regulation of $m s x C$ expression. In addition, we showed that $m s x$ gene expression patterns differ significantly between Xiphophorus and zebrafish. We also included in our study the gonopodium, a testosteronedependent anal fin modification that serves as a fertilization organ in males of live-bearing fishes. Our finding that increased levels of $m s x C$ expression are associated with the testosterone-induced outgrowth of the gonopodium might suggest either that at least parts of the signaling pathways that pattern the evolutionary older gonopodium have been coopted to evolve a sexually selected innovation such as the sword or that increased $m s x C$ expression may be inherent to the growth process of long fin rays in general.

\section{INTRODUCTION}

Secondary sexual characteristics evolve in response to sexual selection. A prominent example for this phenomenon had originally been described by Darwin (1871) in fish of the genus Xiphophorus (Poeciliidae), the so-called swordtails. In males, the ventral caudal fin rays are strongly elongated and often ornamented with bright pigmentation, forming a conspicuous extension of the caudal fin that resembles a sword (Fig. 1). Interestingly, females of the green swordtail, Xiphophorus helleri, and several other species preferentially associate with males carrying a longer sword over males with shorter swords, which is thought to result in a higher mating success of long-sworded males (Basolo 1990a,b). Intriguingly, this behavior is paralleled by the preference of female widowbirds for males with long tail feathers, exemplifying a general trend in Fisherian evolution for exaggerated male traits (Fisher 1930; Andersson 1982).
Within the genus Xiphophorus, several species do not develop a sword, the so-called platyfish. The origin of the sword is debated and relies on a robust phylogeny of the genus, which despite various efforts using morphological, electrophoretic, and molecular characters is still debated in some details (Rauchenberger et al. 1990; Basolo 1991; Meyer et al. 1994; Borowsky et al. 1995; Marcus and McCune 1999). Hybridization experiments between platys and swordtails suggested that several "sword genes" are required to induce a caudal fin extension (Zander and Dzwillo 1969). Although some of these factors might be present in platyfish, in the absence of further sword gene activity, as it is supplied by the swordtail genome in hybrids, fin elongation does not occur (Zander and Dzwillo 1969). However, the molecular mechanisms underlying both phylogenetic and ontogenetic sword formation and putative subsequent sword loss so far have remained completely unknown. 

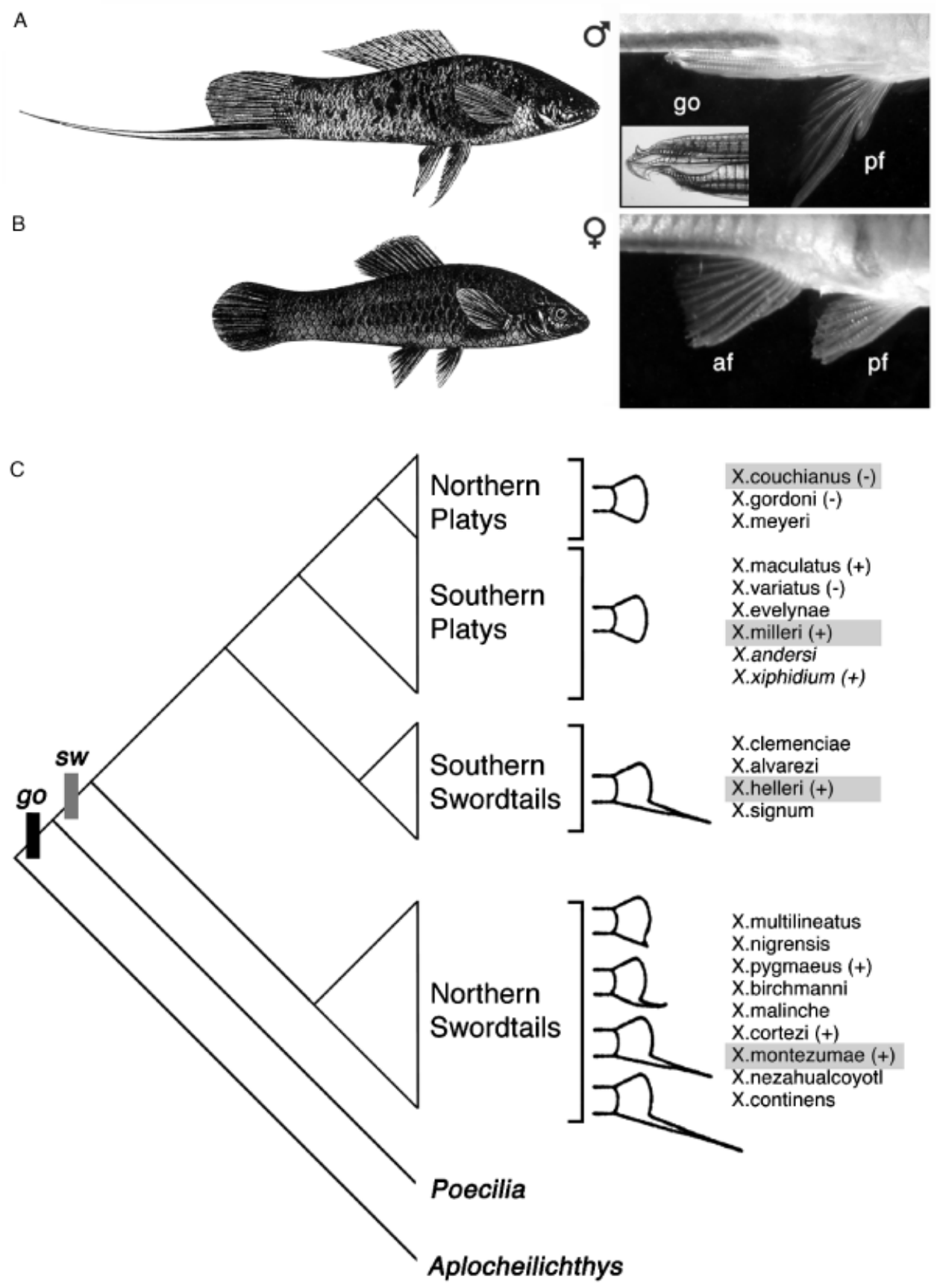

Fig. 1. Fin modifications in Xiphophorus helleri. (A) The adult male features a prominent sword and a gonopodium (magnified in inset), which are sex-specific modifications of caudal and anal fin rays, respectively. (B) The adult female is swordless and has an unmodified anal fin. (C) Consensus taxonomy of platyfish and swordtails, based on a molecular phylogeny (Meyer et al. 1994), to which the traits "sword" (sw) and "gonopodium" (go) are mapped. A gonopodium is present in all Poeciliinae (the subfamily including Xiphophorus and Poecilia species) but is not found in other subfamilies of Poeciliidae, here represented through Aplocheilichthys (Meyer and Lydeard 1993). Typical ranges of phenotypic variation within subgroups are indicated. Species in which caudal fin modifications have been observed upon androgen hormone treatment are marked " $(+)$," and " $(-)$ " if treatments had no effect (Gordon et al. 1943; Zander and Dzwillo 1969). Two species with colorless ventral caudal fin extensions that group within the platys are indicated by italics. Species used in this work are marked by gray background. af, anal fin; pf, pelvic fin. In A and B anterior is to the right. (From Fig. 29 in Darwin 1871).

Juvenile male swordtails are initially swordless. The onset of sexual maturity marks the initiation of the development of secondary sexual characters. A sustained increase of testoste- rone levels during male sexual maturation has been shown to be sufficient to initiate the molecular pathway toward sword development. Moreover, exogenous addition of testosterone 
can induce a caudal fin protrusion in swordtail females, in juvenile males, and some normally swordless platy species (Fig. 1C) (Dzwillo 1962; Zander and Dzwillo 1969). During sword development, the ventral caudal fin rays develop with positive allometric growth by adding additional segments of regular segment length to the distal tip of the fin and develop a characteristic pigmentation pattern. Although the sword is an ornamental fin ray modification specific to the genus Xiphophorus, another male structure derived from anal fin rays, the gonopodium, is a common functional feature of all fishes of the subfamily Poeciliinae (Fig. 1, A and C). The gonopodium arises during sexual maturation in male fish under the influence of testosterone by growth and differentiation of the anterior anal fin rays and serves as a copulatory organ in these live-bearing fish (Grobstein 1942). Nothing is known about the molecular pathways on which androgen hormones act during formation of the sword and the gonopodium.

The study of zebrafish fin development and regeneration has improved knowledge about the mechanisms and genes involved in fin growth and regeneration (Johnson and Bennet 1999). The unpaired teleost fins consist of segmented fin rays, which are formed by pairs of lepidotrichia, U-shaped bones that enclose blood vessels, nerves, pigment cells, and fibroblast-like cells. Fins grow along their proximodistal axis by adding new segments to the distal tips of the fin rays.

The ability of teleosts to regenerate the dermal fin skeleton is gaining increasing attention because of the practical advantages in using zebrafish as a model organism to study the molecular mechanisms underlying growth and regeneration of fins. Teleost fin regeneration can be subdivided into three phases: wound healing, blastema formation, and outgrowth (Johnson and Bennet 1999). The blastema is a mass of undifferentiated cells that later progressively differentiate to form the regenerated tissue. Fibroblast growth factor $(f g f)$ signaling seems to be one of the key processes during blastema formation and regenerative outgrowth (Poss et al. 2000). Fgf receptor 1 ( $f g f r l)$ is expressed in mesenchymal cells proximal to the amputation plane before and during blastema formation, as well as during the outgrowth phase. Blocking of $f g f$ signaling inhibits the formation of a blastema, whereas the process of wound healing seems to be unaffected (Tawk et al. 2002). The $f g f$ signal is believed to originate in the covering epidermis, where a wound $f g f$ has been shown to be expressed (Poss et al. 2000). Together, these gene interactions are believed to control the process of regenerative outgrowth and lepidotrichia formation.

$M s x$ and the closely related $d l x$ genes are expressed during all phases of vertebrate development and mediate patterning and morphogenesis in several tissues, including neural crest cells, limb buds, and the central nervous system (Bendall and Abate-Shen 2000). Msx genes have been shown to act as transcriptional repressors and inhibit differentiation of several cell types (Odelberg et al. 2000; $\mathrm{Hu}$ et al. 2001). During regeneration of the zebrafish fin, several $m s x$ genes are expressed in distinct regions in the regenerating blastema: $m s x A$ and $m s x D$ are transcribed in the wound epidermis, whereas $m s x B$ and $m s x C$ expression is restricted to the distal tip of the blastemal mesenchyme (Akimenko et al. 1995), suggesting a role in epithelium-mesenchyme interactions. Indeed, expression of $m s x A$ and $m s x D$ has been shown to be induced by the distal most blastema (Murciano et al. 2003). The evolutionary relationships between fish and tetrapod msx genes have proven difficult to resolve due to high sequence conservation within the homeobox, which gives little phylogenetic information, and high sequence divergence elsewhere among the proteins (Ekker et al. 1997). The phylogenetic relationships of the five known zebrafish genes to the three tetrapod msx genes remain somewhat tentative, with the exception of the finding that zebrafish $m s x B$ and $m s x C$ are more closely related to one another and to mouse $m s \times 3$ than to other msx genes (Ekker et al. 1997).

In this study, knowledge about zebrafish fin growth and regeneration has been used as a starting point to better understand growth and regeneration of a sexually selected fin modification in the genus Xiphophorus. From a developmental point of view, we asked whether regulation of $m s x$ gene expression, a candidate gene that might be involved in sword development, is associated with growth of the sword during development and regeneration. From an evolutionary point of view, we were interested in whether an understanding of the molecular basis of sword development would help in understanding the evolutionary mechanisms of sexual selection. To address these issues, we cloned candidate $m s x$ genes expressed in regenerating blastemal tissue and showed that $m s x C$ is expressed at higher levels in the sword forming fin rays during a distinct phase of ray outgrowth. In contrast, regulation of $m s x E l$ expression in sword rays is indistinguishable from dorsal fin rays. Moreover, $m s x C$ expression in Xiphophorus surprisingly differs from zebrafish $m s x C$ expression, therefore raising doubts about the validity of zebrafish as a general model to describe the mechanism of fin regeneration in actinopterygians. Possible interpretations of our findings are discussed in the context of the known signaling processes within the blastema. Elucidating the molecular basis of sword formation might help in reconstructing the events of sword gains and losses in the genus Xiphophorus and lead to a better understanding of developmental and genetic mechanisms of sexual selection.

\section{MATERIALS AND METHODS}

\section{Fish stocks and maintenance}

Fish were kept at $24^{\circ} \mathrm{C}$ in densely planted aquaria. Xiphophorus helleri and $X$. couchianus were taken from stocks kept at the 
University of Konstanz, where they are bred randomly. Xiphophorus montezumae and $X$. milleri were kind gifts from Manfred Schartl, University of Würzburg.

\section{Testosterone treatment and amputation}

Up to five individuals of $X$. helleri, aged between 3 and 6 months, were placed in an 8-liter tank. 17 $\alpha$-Methyltestosterone $(1 \mathrm{mg} / \mathrm{ml}$ stock solution in ethanol; Sigma-Aldrich, Munich, Germany) was added to the water twice a week to a final concentration of $10 \mu \mathrm{g} / \mathrm{l}$. For fin amputation, juvenile and adult fish, aged 3-6 months for testosterone treatments or 1-2 years for the remaining experiments, were anesthetized by incubation in a solution of $0.08 \mathrm{mg} / \mathrm{ml}$ tricaine (3-aminobenzoic acid ethyl ester methanesulfonate; Sigma) until pectoral fin movement ceased. Using a sterile razor blade, the first amputation was performed routinely at right angles to the medial caudal fin rays, approximately removing the posterior half of the fin. Subsequently, fins were allowed to regenerate at $24^{\circ} \mathrm{C}$ for variable time periods, depending on the experiment. Fish were anesthetized again and the blastema removed. Amputated blastemata were either fixed in $4 \%$ paraformaldehyde/phosphatebuffered saline (PBS) overnight for subsequent in situ hybridization or alternatively were directly transferred to TRIZOL (GIBCO, Karlsruhe, Germany) for RNA extraction.

\section{RNA extraction and reverse transcription-polymerase chain reaction (RT-PCR)}

TRIZOL $0.5 \mathrm{ml}$ was added to up to nine blastemata immediately after amputation. The sample was incubated at room temperature for $5 \mathrm{~min}$ and then vortexed vigorously for several minutes. All subsequent steps were performed following manufacturers' instructions. Total RNA was eluted in $50 \mu$ diethyl pyrocarbonate (DEPC)-treated water. Single-stranded complementary DNA was synthesized from total RNA using the SuperscriptII system (GIBCO-BRL) according to manufacturer's instructions. Before PCR experiments, single-stranded complementary DNA was purified using a PCR purification kit (Qiagen, Hilden, Germany) and eluted in $30 \mu \mathrm{l}$ elution buffer. Primers were designed using alignments of zebrafish $m s x$ genes and fugu genomic sequence information (www.jgi.doe.gov/fugu) with high similarity to the latter. A 475-bp $m s x E$ fragment (GenBank accession no. AY150557) was amplified by using primers msx-fwd2: $5^{\prime}$ CCGTTCAGCGTGGAG- $3^{\prime}$ and msx-rev2: 5'-TCCTGGAGCCGCTTGG-3' ${ }^{\prime}$ under the following conditions: $2 \mathrm{~min}$ $94^{\circ} \mathrm{C}$, then 40 cycles of $30 \mathrm{sec} 94^{\circ} \mathrm{C}, 1 \mathrm{~min} 52^{\circ} \mathrm{C}, 1 \mathrm{~min} 72^{\circ} \mathrm{C}$. A 635-bp msxC fragment (GenBank accession no. AY150556) was amplified by using primers msx-fwd4: 5'-CTCCCGTTCAGCGTCG-3' and msx-rev3: 5'-TAAGACAAATAATACATCCC $-3^{\prime}$ under the following conditions: $2 \mathrm{~min} 94^{\circ} \mathrm{C}$, then 35 cycles of $30 \mathrm{sec} 94^{\circ} \mathrm{C}, 30 \mathrm{sec} 47^{\circ} \mathrm{C}, 30 \mathrm{sec} 72^{\circ} \mathrm{C}$. In both cases a final extension step of $7 \mathrm{~min}$ at $72^{\circ} \mathrm{C}$ was added. PCR products were gel purified using a kit (Qiagen) and cloned into the pCR2.1-TOPO vector (Invitrogen, Karlsruhe, Germany) for sequencing.

\section{In situ hybridization and histology}

For in vitro transcription, inserts were cloned into $\mathrm{pBKS}$ using Xho $\mathrm{I}$ and BamHI $(m s x C)$ and Xho I and HindIII ( $m s x E)$ restriction sites, respectively. In situ hybridization was performed as described
(Begemann and Ingham 2000) with $10 \mathrm{~min}$ of proteinase $\mathrm{K}$ treatment at a final concentration of $14 \mu \mathrm{g} / \mathrm{ml}$ before prehybridization. All in situ experiments on regenerating or testosteroneinduced primary growing fins were performed with a minimum total number of three individuals, all showing comparable results. Sense probes, which initially were applied in parallel to anti-sense probes, never stained above background level. Because of the high sequence conservation within the genus, $X$. helleri antisense probes could also be used in the swordtail $X$. montezumae and in the platyfish $X$. milleri and $X$. couchianus.

For longitudinal sections, stained fins were incubated over night in $10 \%$ formic acid and subsequently enclosed in an agarosesucrose gel (1.5\% agarose, 5\% sucrose in PBS). Solidified agar blocks were trimmed and placed in a solution of $30 \%$ sucrose in PBS over night. Agar blocks were covered with tissue-embedding medium. Cryosections $(25 \mu \mathrm{m})$ were mounted on microscope slides and covered with $70 \%$ glycerol in PBS for photography.

\section{Phylogenetic analysis}

Deduced amino acid sequences of the obtained fragments were aligned to zebrafish and other tetrapod $m s x$ genes using CLUSTAL $\mathrm{W}$ as supplied by the BIOEDIT software package (North Carolina State University). Sequences of Mus, Xenopus, and Danio were identical to those in Ekker et al. (1997). Fugu sequences for putative $m s x C$ (scaffold 1488) and $m s x E / 1$ (scaffold 12) were obtained from the Fugu genome project (release 17_05_02; Aparicio et al. 2002). Neighbor-joining phylogenies were constructed with the TREECON software package (Van de Peer and De Wachter 1993), using KIMURA83 distances and 1000 bootstrap iterations.

\section{RESULTS}

\section{Cloning and phylogenetic analyses of two Xiphophorus msx genes}

To gain a better understanding of the molecular mechanisms governing growth and differentiation of the caudal fin and its ventral modification, the sword, in the green swordtail, Xiphophorus helleri, we used an RT-PCR strategy to amplify $m s x$-related sequences. Reasoning that many genes important for larval fin development may also be expressed during regeneration after fin amputation, we used regenerating caudal fin blastemata as a source of RNA. Two fragments were amplified, both of which extend in the $3^{\prime}$ direction from the end of a conserved decapeptide characteristic for $m s x$ genes (typically LPFSVEALMA) (Ekker et al. 1997). A 635bp fragment included the homeobox and the $3^{\prime}$ end of the coding sequence, whereas a 475 -bp fragment terminated near the end of the homeobox.

To determine the relationships between these new $X$. helleri gene family members and known genes, we aligned their deduced amino acid sequences to perform a phylogenetic analysis (Fig. 2A). The resulting tree (Fig. 2B) identified the 635- and 475-bp fragments of $X$. helleri as Orthologs of 
A

msxC-Xiphophorus msxC-Fugu msxC-Danio msxB-Danio msx 3-Mus msxD-Danio msxa-Danio msx2 - Xenopus msx2-Mus msxE/1-Xiphophorus msxE/1 - Fugu msxE-Danio msx 1 -Xenopus msx1-Mus

msxC-Xiphophorus msxC-Fugu msxC-Danio msxB-Danio $\mathrm{msx} 3$ - Mus msxD-Danio msxA-Danio msx2 - Xenopus $\mathrm{msx} 2$-Mus msxE/1-Xiphophorus msxE/ 1 - Fugu msxE-Danio msx1-Xenopus msx1-Mus

msxC-Xiphophorus msxC-Fugu msxC-Danio msxB-Danio msx3-Mus msxD-Danio msxA-Danio msx2 - Xenopus msx2 - Mus msxE/1-Xiphophorus msxE/1-Fugu msxE-Danio msx1-Xenopus msx1-Mus

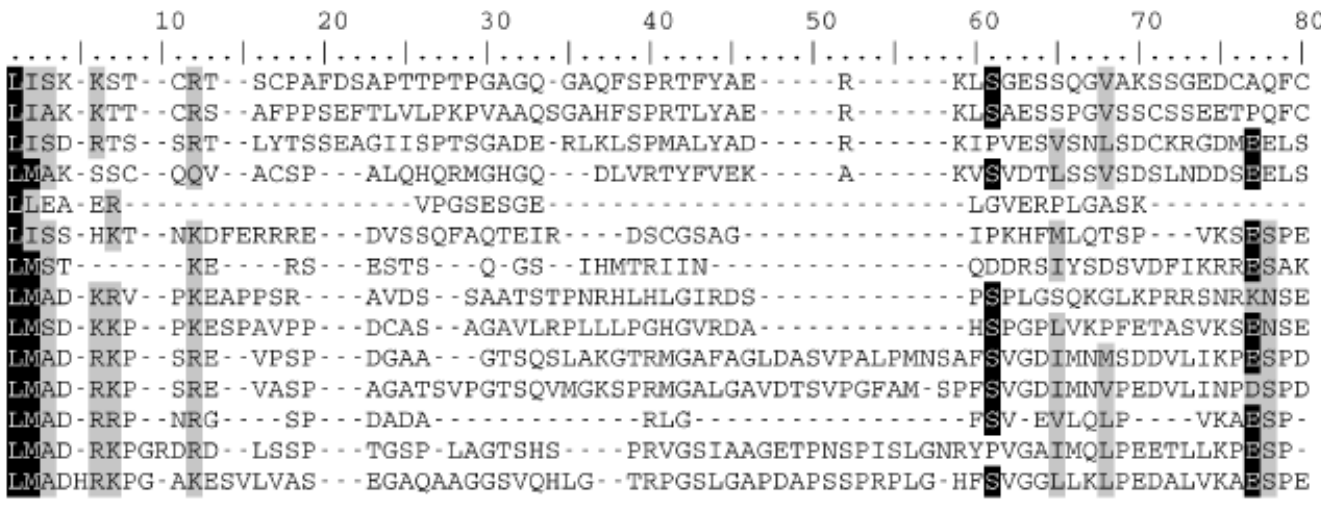

90
$\ldots|\ldots| \ldots|\ldots| \ldots \mid$

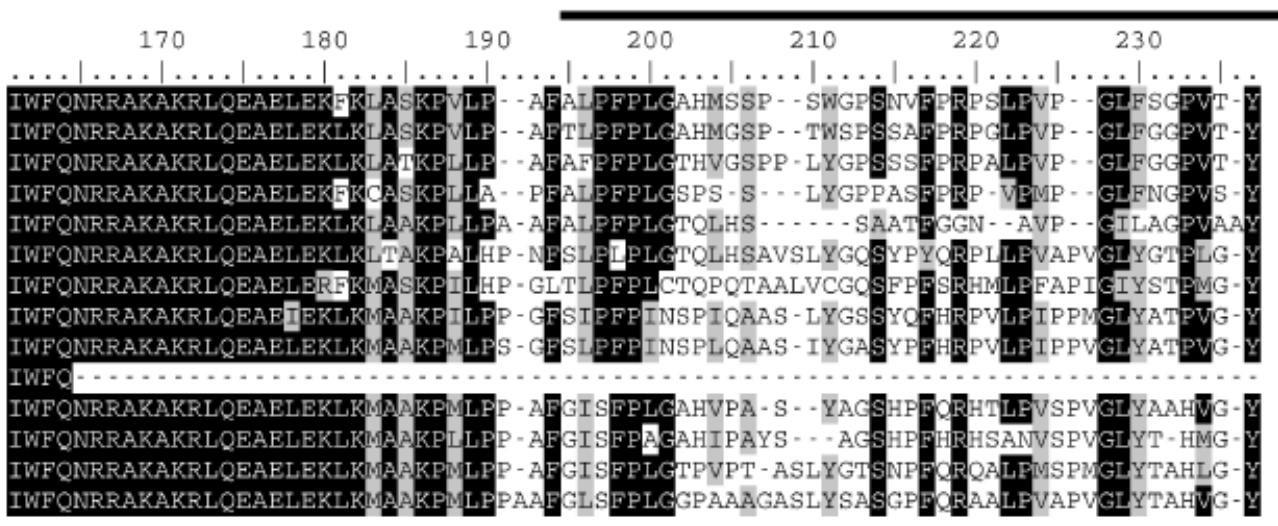

B

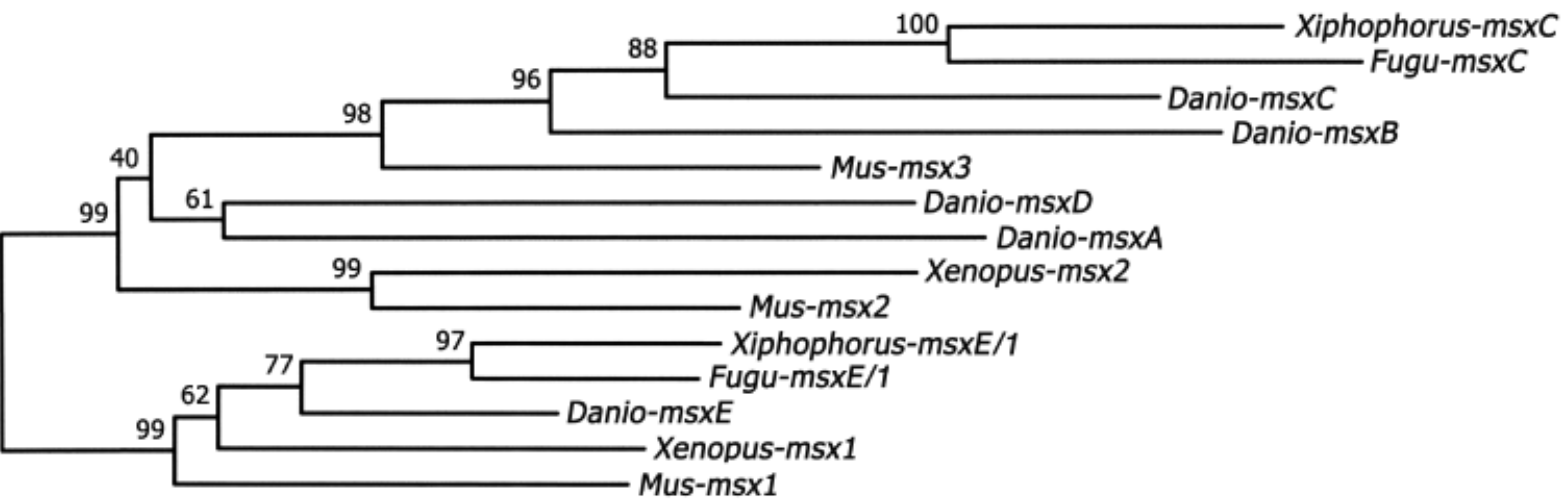


zebrafish $m s x C$ and $m s x E$, respectively. However, because the alignment of the complete available sequences contained many poorly conserved positions, we also aligned smaller portions of the two Xiphophorus sequences (spanning the homeodomain and neighboring regions) separately with their putative orthologs and constructed neighbor-joining trees (data not shown). The phylogenetic position of the 635-bp fragment remained unchanged in this analysis, whereas the 475-bp fragment appeared to be more closely related to Xenopus msxl than to Danio msxE in the tree based on the shorter alignment. Consistent with this finding is the observation that the homeodomain was conserved between the Xiphophorus sequence and Xenopus msxl but showed three amino acid exchanges when compared with Danio msxE. We therefore refer to the Xiphophorus and Fugu sequences as $m s x E / 1$ as long as the relationships of tetrapod $m s x 1$ and fish $m s x E$ are not solved (Ekker et al. 1997) and to the second clone as $\operatorname{msxC}$.

\section{Expression of $\boldsymbol{m s x} \boldsymbol{C}$ in developing swordtail fins}

We determined the expression patterns of $m s x C$ and $m s x E / 1$ during growth of caudal and anal fins of $X$. helleri. To compare expression levels between growing sword rays and neighboring nonsword fin rays, $m s x C$ expression was studied in caudal fins with a small growing sword of 4- to 6-monthold maturing male fish. During primary caudal fin growth, msx $C$ was expressed at the distal tip of all fin rays (Fig. 3A). Fin rays that contributed to the sword exhibited markedly higher expression levels of $m s x C$ than the remaining nonsword fin rays (Fig. 3B).

The growth of the sword and gonopodium is a result of sexual maturation under the influence of male sex hormones. To test if sword growth is generally correlated with upregulation of $m s x C$ expression, we studied $m s x C$ expression in testosterone-induced sword rays. After 10 days of testosterone treatment, sexually immature fish developed a small ventral protrusion of the caudal fin of approximately $2 \mathrm{~mm}$ in length (Fig. 3C). Like in naturally growing fins, $m s x C$ is strongly expressed in the distal tips of sword rays of the caudal fin (Fig.

Fig. 2. Amino acid alignment and phylogenetic analysis of partial sequences of two Xiphophorus helleri msx genes. (A) Amino acid alignment of partial sequences of 14 vertebrate $m s x$ genes, starting at the end of a conserved decapeptide. Identical and similar amino acids are shaded black and gray, respectively, if they appear in at least $60 \%$ of the sequences. The homeodomain is indicated by a black bar. (B) Neighbor-joining tree based on the alignment in A. The novel Xiphophorus sequences cluster with Danio rerio msxC and $m s x E$, respectively. However, because the orthologous relationships between fish $m s x E$ and tetrapod $m s x l$ are not solved, the Xiphophorus and Fugu sequences are assigned as $m s x E / 1$ (for details see text and Ekker et al. 1997). Numbers indicate bootstrap values.
3D), whereas expression in more dorsal rays is weak at best (Fig. 3E).

The high levels of $m s x C$ expression observed in growing sword rays could be either a specific feature of testosteroneinduced growth or generally correlated with growth of fin rays. To address these possibilities, we analyzed $m s x C$ expression in caudal fin rays of $X$. helleri larvae at 1 day after birth (Fig. 3F). Growth of the larval caudal fin is associated with high levels of $m s x C$ expression, whereas $m s x E / 1$ is expressed at very low levels during primary fin growth (not shown). We proposed that although expression of $m s x C$ is a general feature of growing fin rays, expression of $m s x C$ is particularly up-regulated upon both endogenous and artificial induction of sexual maturation in fin rays that participate in sword formation.

Another structure generated upon sexual maturation under the influence of testosterone is the gonopodium, which forms by growth and differentiation of a subset of anterior fin rays of the anal fin. We analyzed $m s x$ gene expression in testosterone-induced gonopodia of immature $X$. helleri of undefined genetic sex after 1, 2, and 3 weeks of treatment. Because it was shown that younger fish are more responsive to testosterone and upon treatment will develop a more typical gonopodium (Grobstein 1942), immature fish were chosen for this study instead of mature females. After 1 week of testosterone treatment, $m s x C$ was first up-regulated in anal fin rays $3-5$, the so-called $3,4,5$ complex, which comprise the gonopodium (Fig. 3, G and H) (Grobstein 1942; Hopper 1949; Angus et al. 2001). After 2 weeks the length of the gonopodial rays exceeded that of neighboring anal fin rays by approximately $1.5 \mathrm{~mm}$, and $m s x C$ continued to be expressed in deeper mesenchymal cells at the edge of the gonopodium (Fig. 3H). Unlike in larval fins and growing swords, expression is not restricted to the distal tips of the fin rays but forms a continuous stripe of expression at the caudal edge of the gonopodium (Fig. $3 \mathrm{H}$ ). After 3 weeks of treatment only faint expression is detected in the gonopodium (not shown), whereas untreated fins do not express $m s x C$ (Fig. 3I).

\section{Expression of $m s x C$ and $m s x E / 1$ in regenerating swordtail caudal fins}

The comparison of expression levels between sword rays and more dorsal rays can be misleading during primary sword growth, because at that time sword rays grow at a substantially faster rate compared with dorsal rays. The examination of regenerating fins allowed a detailed comparison of growth control mechanisms of normal and sword fin rays simultaneously in a single individual. Despite clear differences between the processes of fin development and regeneration (Akimenko et al. 1995), it is assumed that the general mechanisms of growth control that act during larval development are reestablished during regeneration (Laforest 

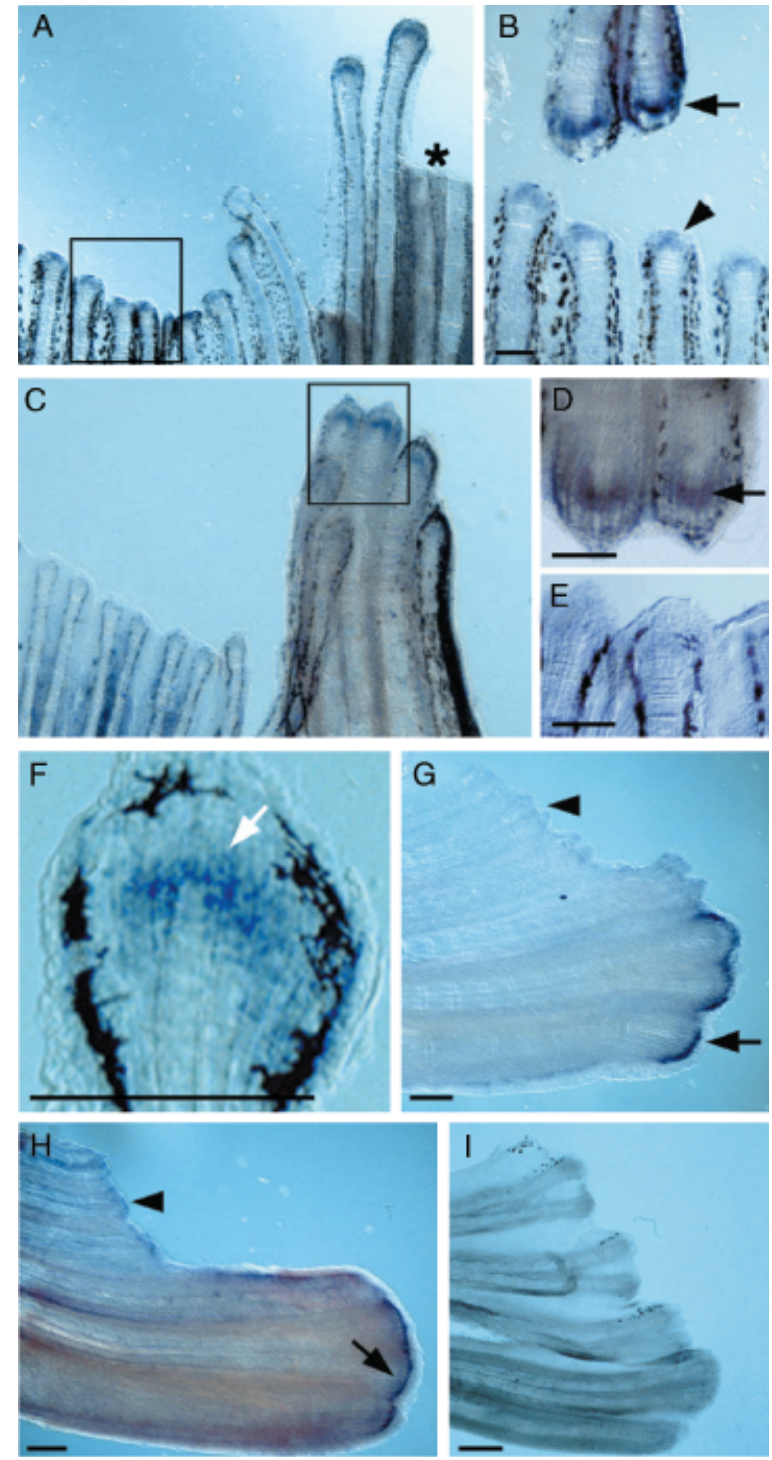

Fig. 3. Expression of $m s x C$ in developing fins of Xiphophorus helleri. (A and B) msxC is expressed at low levels in the tips of growing nonsword rays of a maturing animal aged 4-6 months (box; arrowhead in B), whereas expression levels are high in sword rays of the same fin (arrow in B). Asterisk marks original position of sword rays shown in B. (C-E) Testosterone treatment of immature $X$. helleri induces sword outgrowth and strong ms $x C$ expression in sword rays (box; arrow in D); nonsword rays of the same fin (C and E) express below the level of photographic detection. (F) $M s x C$ is expressed in the tip of developing larval fins (1 day after birth). ( $\mathrm{G}$ and $\mathrm{H}) \quad M s x C$ expression (arrows) in testosterone-induced growing gonopodia after 1 week $(G)$ and 2 weeks $(\mathrm{H})$ of treatment. Arrowheads mark low level expression in nongonopodial rays. (I) Untreated anal fins do not express $m s x C$ at significant levels. (A, C, E, F) Posterior is on top, ventral is to the right; (G-I) ventral is to the right, anterior is to the bottom. Bar, $100 \mu \mathrm{m}$. et al. 1998; Borday et al. 2001). To analyze the expression domains of $m s x C$ and $m s x E / 1$ during fin regeneration, we compared swordless adult female fin blastemata 5 days after amputation in $X$. helleri, using one half of each fin for $m s x C$ and the other half for $m s x E / 1$ staining, respectively. In contrast to zebrafish, where $m s x E$ expression is not detectable in regenerating fins (Akimenko et al. 1995), Xiphophorus $m s x E / 1$ is expressed in the distal-most blastema of regenerating swordtail fins (Fig. 4B). $M s x C$ expression is found in two domains, in the distal-most blastema and in a more proximal region of the regenerating tissue (Fig. 4A). Both genes are expressed in mesenchymal cells of the blastema itself and are absent from the wound epithelium (Fig. 4, C and D). Most interestingly, $m s x C$ is further expressed in a proximal domain of lateral mesenchymal cells beneath the wound epidermis, an expression domain that has not been reported in zebrafish. We were further unable to detect this $m s x C$ expression domain
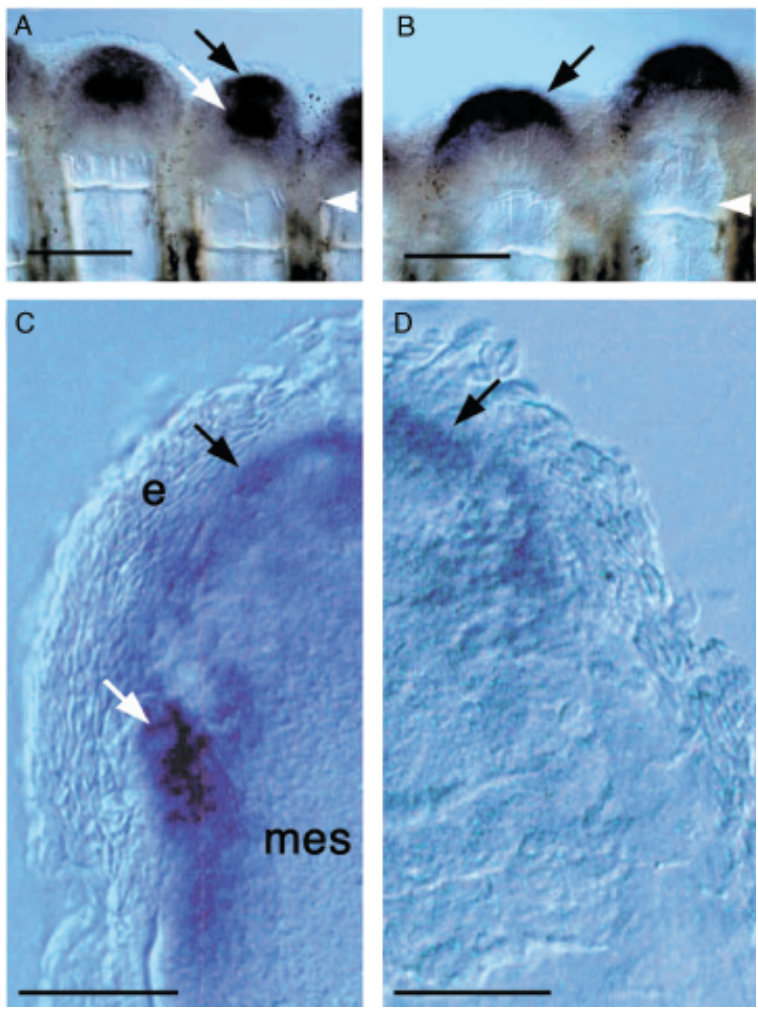

Fig. 4. Expression of $m s x C$ and $m s x E$ in regenerating fins of Xiphophorus helleri (5 days after amputation). (A and C) $M s x C$ is expressed in two domains, in the distal tip of the blastemal mesenchyme (mes; black arrows) and more proximally in the mesenchyme, abutting the epidermis (e; white arrows). (B and D) $M s x E / 1$ is expressed exclusively in the distal tip of the blastemal mesenchyme. (A and B) Whole mounts of the same fin; arrowheads indicate level of amputation; posterior is on top, ventral is to the right. (C and D) Longitudinal sections; posterior is on top. Bars: A and $\mathrm{B}, 150 \mu \mathrm{m} ; \mathrm{C}$ and $\mathrm{D}, 50 \mu \mathrm{m}$. 
in zebrafish fin regenerates (data not shown). Based on comparing $m s x$ gene expression with published analyses of fin regeneration, we infer that expression of both $m s x C$ and $m s x E$ / $l$ in the distal mesenchyme of regenerating fin rays colocalizes with undifferentiated pluripotent blastemal cells, whereas expression of $m s x C$ in the lateral mesenchyme coincides with the position of cells confined to become scleroblasts.

To address the question of whether $m s x C$ and $m s x E / 1$ play a role in growth control during regeneration of sword and nonsword fin rays, we analyzed gene expression in $X$. helleri
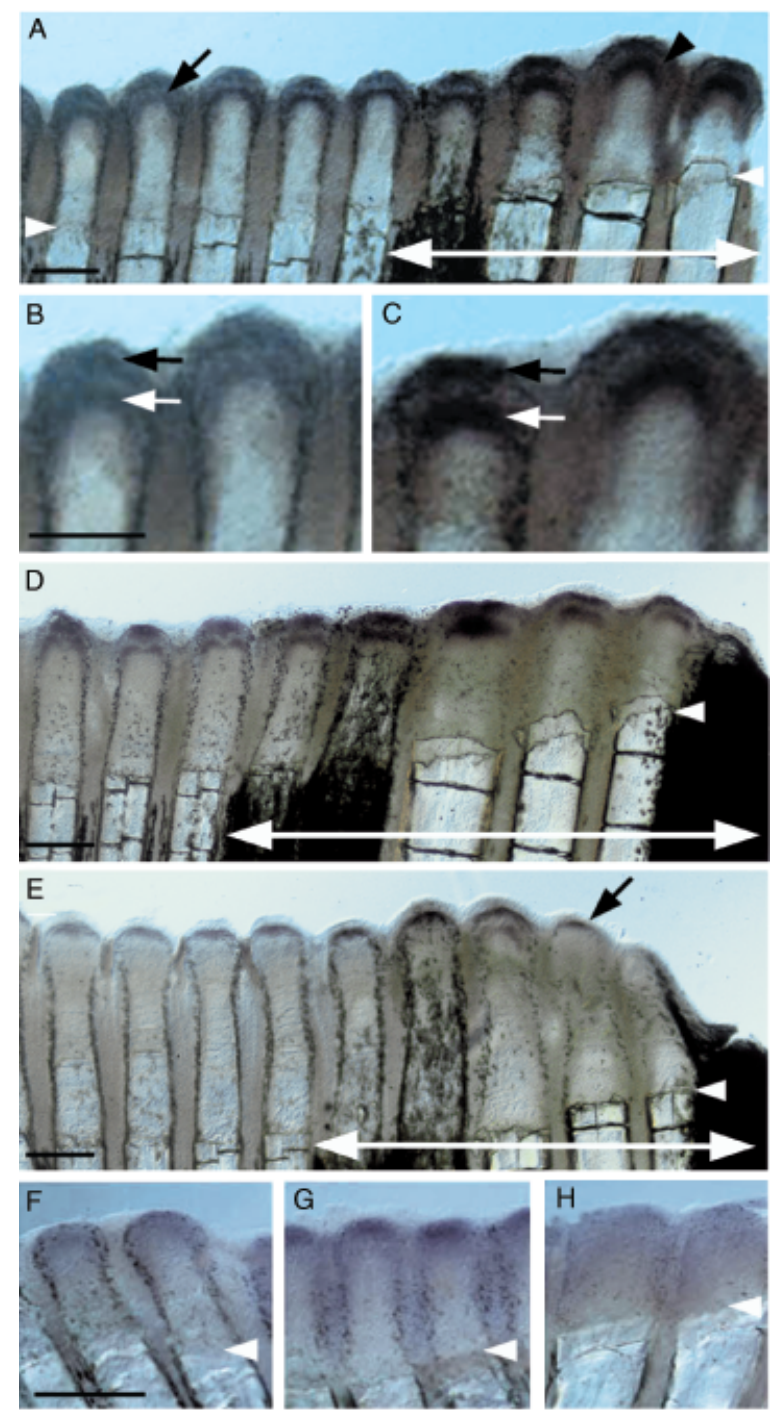

I

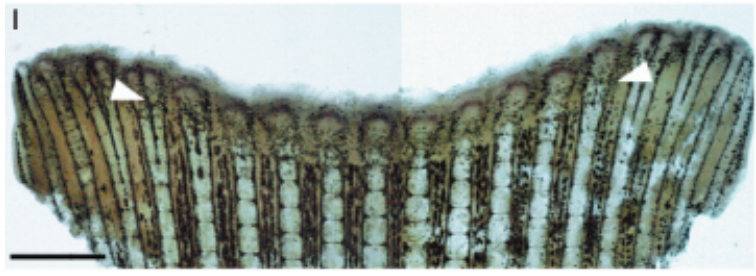

male caudal fin blastemata at different times after amputation. In young blastemata of up to 4 days after amputation, we observed no apparent differences in $m s x C$ expression levels by visual inspection (not shown). However, from 5 to 6 days after amputation onward, high levels of $m s x C$ are still detectable in sword rays, compared with nonsword rays (Fig. 5, A-C), where expression levels decreased substantially. Beginning at 7 days after amputation, $m s x C$ expression in the proximal domain starts to drop below detectable levels after around 9 days after amputation (Fig. 5, D and E); at this stage, the expression in the distal-most mesenchyme is still present. In contrast to $m s x C$, no up-regulation of $m s x E / 1$ in sword ray blastemata could be detected at any stage up until 7 days of regeneration. In fact, $m s x E / 1$ is consistently expressed at very low levels in rays that are close to the dorsal or ventral margins of the fin (Fig. 5, F and $\mathrm{H}$ ), whereas expression in medial rays appears to be stronger (Fig. 5G).

Finally, if elevated levels of $m s x C$ expression are associated with regenerating sword rays, then all fin rays of male platyfish should express similar levels of the gene. We tested this hypothesis on a northern ( $X$. couchianus) and a southern (X. milleri) platy (Fig. 1C) and could not detect higher $m s x C$ expression in ventral sword rays of either species (Fig. 5I and not shown).

We conclude that both in development and during regeneration high expression levels of $m s x C$ are associated with a subset of fin rays that will grow for an extended period and differentiate into male-specific structures. Taken together, our analyses identify $m s x C$ as a gene whose transcriptional regulation is correlated with final fin ray fate in swordtails.

\section{Comparison of $m s x$ expression profiles in $X$. helleri and $X$. montezumae}

To investigate whether differential regulation of $m s x C$ expression in sword forming fin rays is a general feature of

Fig. 5. Time course of $m s x C$ and $m s x E / 1$ expression in regenerating normal and sword rays of Xiphophorus helleri males. (A-C) At 6 days after amputation $m s x C$ is expressed in both distal blastema and lateral mesenchyme at higher levels in sword rays (black arrowhead) than in nonsword rays (black arrow). (B and C) Magnification of representative sword (C) and nonsword (B) rays, with distal (black arrow) and proximal (white arrow) expression domains. (D) At 7 days after amputation, $m s x C$ expression starts to cease in the sword rays. (E) At 9 days after amputation, $m s x C$ expression starts to be lost in the proximal mesenchymal domain. $(\mathrm{F}-\mathrm{H}) \mathrm{Ms} x E / 1$ is expressed at slightly higher levels in blastema of medium rays $(\mathrm{G})$ as compared with dorsal $(\mathrm{F})$ and sword $(\mathrm{H})$ rays of the same fin (5 days after amputation). (I) In males of the platyfish $X$. couchianus, msxC is expressed at similar levels in all regenerating fin rays (5 days after amputation), irrespective of position. Arrowheads indicate level of amputation; white double arrows indicate sword rays. Posterior is on top, ventral is to the right. Bars: A, B, D-F, $100 \mu \mathrm{m} ; \mathrm{I}, 300 \mu \mathrm{m}$. 


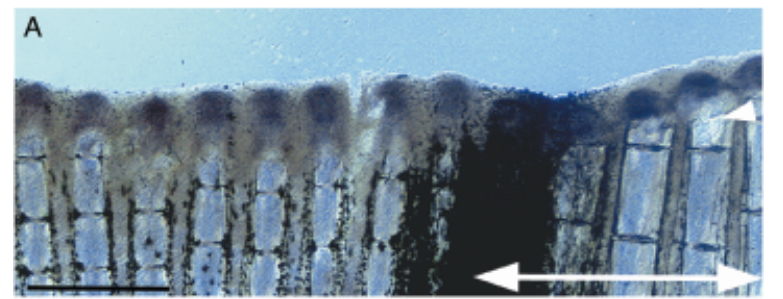

B
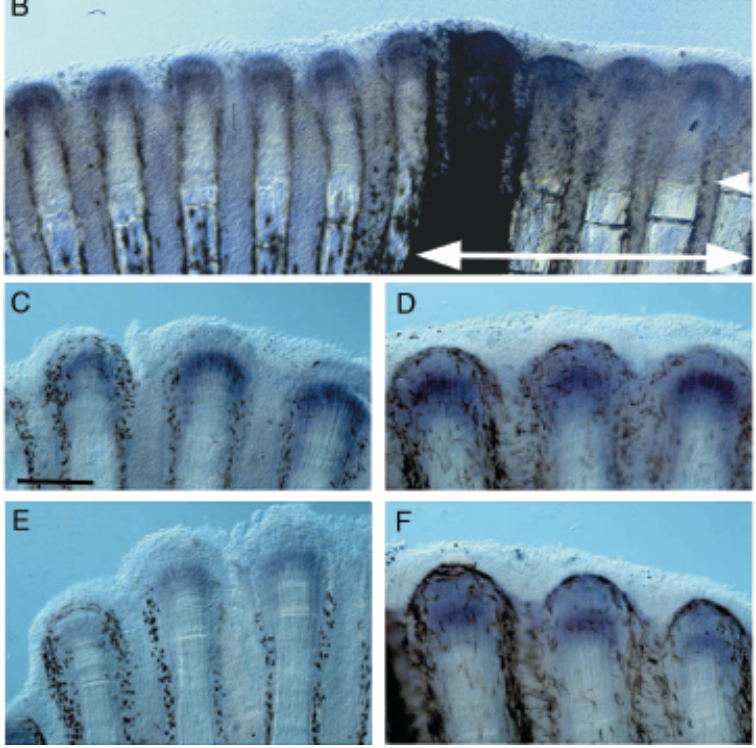

Fig. 6. Time course of $m s x C$ expression in regenerating normal and sword rays of Xiphophorus montezumae. (A and B) During early regenerative outgrowth (A, 4 days after amputation; B, 6 days after amputation) no higher levels of $m s x C$ could be detected in sword rays compared with normal rays. (C-F) At 7 days after amputation $m s x C$ expression ceases in nonsword rays (C) but persists in sword rays (D) of the same fins. (E and F) At 9 days after amputation expression in sword rays $(\mathrm{F})$ is much stronger than in nonsword rays (E). White double arrows indicate sword rays. Posterior is on top, ventral is to the right. Bars: A, $400 \mu \mathrm{m} ; \mathrm{C}$, $100 \mu \mathrm{m}$

sword ray development and whether there is species-specific variation, we compared $m s x C$ expression patterns during fin regeneration between representatives of northern $(X$. montezumae) and southern $(X$. helleri) swordtails. The two species represent distinct clades (Rosen 1979; Rauchenberger et al. 1990; Meyer et al. 1994), each comprising several species with various sword lengths and bear the longest swords within their clades, respectively.

As in $X$. helleri, in $X$. montezumae the blastema takes longer to form in sword rays than in nonsword rays. Until 6-7 days after amputation, no higher levels of $m s x C$ could be detected in sword rays compared with normal rays in $X$. montezumae (Fig. 6, A and B). From 7 days after amputation on we observed lower levels of $m s x C$ expression in nonsword blastemata (Fig. 6, C and E), whereas transcripts were present at high levels in outgrowing rays of the sword (Fig. 6, D and F). Thus, although $m s x C$ is up-regulated in
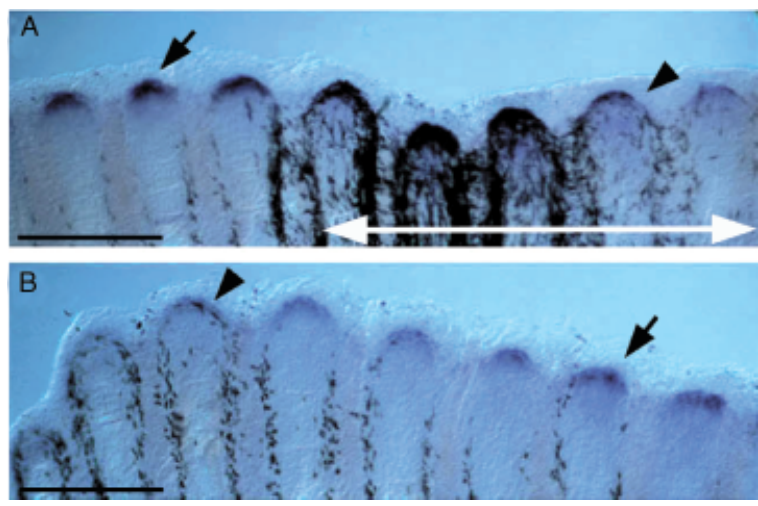

Fig. 7. MsxE/1 expression in blastemata of Xiphophorus montezumae at 9 days after amputation. (A) Ventral caudal fin rays, including the sword region (white double arrows). (B) Dorsal rays. Expression is strong in medium rays (arrows) but weak in blastemata of sword rays and dorsal rays (arrowheads in A and B). Posterior is on top, ventral is to the right. Bar, $200 \mu \mathrm{m}$.

regenerating sword rays in both clades of swordtails, the timing of the process is slightly different, such that the phase of higher $m s x C$ expression in sword rays appears earlier in $X$. helleri than in $X$. montezumae. As in $X$. helleri, $m s x E / 1$ is expressed at very low levels in sword rays at the same time point when $m s x C$ is markedly up-regulated (Fig. 7, A and B). Together our results suggest a role for $m s x C$ in growth control of the male-specific testosterone-dependent fin transformations in the Xiphophorus species examined in this study.

\section{DISCUSSION}

\section{High expression levels of $m s x C$ correlate with sword growth and regeneration}

Sexual selection can lead to the evolution of exaggerated traits that are exclusively present in males. In fish, the genus Xiphophorus provides the opportunity to study the development of such a trait, the caudal fin extension in swordtails, within a microevolutionary framework. In a first approach to identify candidate factors involved in the sword forming process, we characterized the expression of two msx homeobox genes during Xiphophorus fin growth and regeneration. Msx gene expression has been well documented during regenerative growth in the zebrafish fin and in other model organisms (Akimenko et al. 1995; Bendall and Abate-Shen 2000). Moreover, msx genes have been shown to act as inhibitors of differentiation in vitro and in vivo and might therefore be important for regulating continued growth and inhibiting differentiation (Odelberg et al. 2000; Hu et al. 2001). We investigated whether the expression of Xiphophorus $m s x$ genes is associated with the formation of the sword and find that $m s x C$ expression is elevated in growing fin rays of the sword and the gonopodium, both of which respond to 
male sex hormones by an enhanced growth rate. This strong expression is indicative of a general role for $m s x C$ in fin transformations that are associated with reaching sexual maturity.

Time course expression analyses in $X$. montezumae and $X$. helleri caudal fin regenerates reveal that $m s x C$ is expressed at equally high levels in all rays during initial phases of regeneration. However, from 7 to 9 days (X. montezumae) after amputation, expression persists at a high level in sword blastemata but is down-regulated in nonsword rays, suggesting a specific role for $m s x C$ in the formation of sword rays. In agreement with this finding, we did not observe elevated $m s x C$ expression in ventral rays of the caudal fins of swordtail females or male platyfish. In contrast to this, $m s x E / 1$ is not differentially expressed in sword and nonsword rays but is detected at high levels in medium rays, which constitute the center of the caudal fin and only weakly in ventral and dorsal fin rays. From this we conclude that expression in the mesenchymal blastema of sword rays is not a feature of $m s x$ genes in general but appears to be specific at least to $\operatorname{msxC}$.

Because up-regulation of $m s x C$ is observed in developing and in regenerating sword rays of two species of swordtails, it may constitute a general feature associated with sword development. Depending on the inclusion of further characters, such as pigmentation, into the definition of what constitutes a sword, the trait may have arisen several times independently within the genus Xiphophorus (Basolo 1990a, 1991; Meyer et al. 1994; Wiens and Morris 1996; Meyer 1997). Both traditional and molecular phylogenies agree that northern and southern swordtails are members of distinct clades within the genus. Our comparison of $m s x C$ and $m s x E / 1$ expression patterns in $X$. helleri, a southern swordtail, and $X$. montezumae, a northern swordtail, thus addressed the possibility that molecular pathways of sword formation might differ between these groups, if the trait arose independently. Our results point toward similar modes of $m s x C$ transcriptional regulation during sword growth in the two clades. Assuming that tight regulation of $m s x C$ expression is a trigger for male-specific fin modifications, this may indicate that swords evolved only once in the common ancestor of $X$. helleri and $X$. montezumae and were lost repeatedly, as was suggested based on a molecular phylogeny (Meyer et al. 1994).

However, another possibility is that prolonged expression of $m s x C$ is a general feature associated with fin rays that overgrow the remaining rays. If this were the case, the function of $m s x C$ could be either to regulate the identity of ray segments along the proximodistal axis or to promote proliferation. In the first scenario, different expression levels of $m s x C$ confer positional information along the proximodistal axis of all fin rays, such that high and low expression levels determine proximal and distal fates, respectively. Regarding final ray length, regenerating segments in growing rays of the sword, which eventually develop more segments of normal length, would have a more proximal character than segments in nonsword rays at a comparable blastemal position. A prediction of this model is that $m s x C$ would be expressed at a higher level near the base of a fin than in more distal parts. Such a graded expression profile has been reported for $m s x B$ in zebrafish caudal fin blastemata after amputations at various proximodistal levels, with cells of the proximal blastema expressing higher levels than the cells of the distal blastema (Akimenko et al. 1995), but was not observed for other $m s x$ genes, including $m s x C$. Similarly, our preliminary studies on Xiphophorus fins did not detect significantly higher levels of $m s x C$ in proximal blastemata than in distal ones (data not shown).

In the second scenario, $m s x C$ regulates blastemal proliferation rates, such that cells with prolonged $m s x C$ expression proliferate rapidly. In this view, high $m s x C$ expression levels would be a feature common to the strongly growing fin rays in swords and gonopodia. Accordingly, the sword must have arisen through a mechanism independent of $m s x C$ expression. An examination of the consequences of $m s x C$ overexpression, combined with a detailed study of cell proliferation in sword and nonsword rays, will be required to find answers to these questions.

\section{Sword genes and the development of a sexually selected male character}

The term "sword genes" had been introduced for a set of as yet unknown genes whose regulated activity is required for the developmental outgrowth of ventral caudal fin rays in the genus Xiphophorus, ranging from rather small and unpigmented protrusions to long and characteristically pigmented swords (Zander and Dzwillo 1969; Meyer et al. 1994). Common to all such fin modifications is the ability to induce their development through endogenous or exogenous activity of androgen hormones. It should be noted, however, that sword genes are not necessarily restricted in their activity to the developing male fins but that such genes may play additional important roles in other tissues and in various other stages of development. Our results demonstrate that high expression levels of $m s x C$ are associated with primary and regenerative growth of ventral caudal fin rays in two swordtail species within the genus Xiphophorus. Moreover, transcriptional up-regulation of $m s x C$ is a consequence of fin ray elongation in testosterone-induced swords. However, a classification of the $m s x C$ genes in the swordtails $X$. helleri and $X$. montezumae as candidate sword genes would be premature without further knowledge about their actual function in the sword-forming process.

Interestingly, strong $m s x C$ expression is a feature of both sword rays and gonopodial rays. Clearly, the gonopodium is evolutionary older than the sword (Meyer and Lydeard 1993); therefore, a hypothetical scenario for the evolution of the 
sword could be proposed where at least some components of the molecular pathway involved in the development of the gonopodium have been used for sword formation. An alternative scenario would be that delayed down-regulation of $m s x C$ is a general mechanism associated with control of ray length, irrespective of its employment in sword and anal fin ray development.

MsxC is an evolutionary conserved transcription factor with specific functions in several other tissues throughout development. Its contribution to quantitative morphological variation found in these male-specific traits may be possible because of a high spatial and temporal specificity of gene regulation, which may have brought expression of this gene in male-specific fin modifications under direct or indirect control by androgen hormones. Our results can serve as a starting point toward a comparative dissection of the pathways that form the sword and gonopodium within the genus Xiphophorus and warrant further investigations that aim to identify both the molecular targets and upstream regulatory factors of $m s x C$ that are involved in changes of caudal fin regulatory pathways during the evolution of the sword.

\section{Msx genes in teleost fin development and regeneration}

Within regenerating tissue, $m s x B$ and $m s x C$ are exclusively detected in the distal-most blastema, a region of reduced proliferation rate compared with more proximal areas of the blastema (Akimenko et al. 1995; Santamaria et al. 1996; Nechiporuk and Keating 2002). Surprisingly, we find striking differences in expression for both $m s x C$ and $m s x E / 1$ between zebrafish and Xiphophorus. First, msxE is not expressed in regenerating caudal fin blastemata of the zebrafish, and second, we detected Xiphophorus msxC not only in the distal tip of the blastemal mesenchyme, as in zebrafish, but in addition in proximolateral regions of the blastema. This domain is in close vicinity to a region which is known to express $f g f r l$ in zebrafish (Poss et al. 2000). Because $m s x$ genes are believed to be under the control of $f g f$ signaling (Wang and Sassoon 1995), it is tempting to speculate that a signal from the neighboring epidermis is controlling $m s x C$ expression in the lateral mesenchyme. The role of $m s x C$ in this region could be to maintain the undifferentiated state of blastemal cells, which are later differentiating into lepidotrichia forming osteoblasts. However, expression of either zebrafish $m s x C$ or any other zebrafish $m s x$ gene in the lateral mesenchyme remains to be shown. Thus, either in zebrafish another not yet discovered gene has a similar function in the lateral mesenchyme as msxC in Xiphophorus or the process of regulating blastemal growth and differentiation is different in these two rather distantly related species. Our findings suggest that the mechanism of fin regeneration is variable within actinopterygians such that valuable insights into the process might be gained by future examinations of other components of the $f g f$-signaling cascade in Xiphophorus and other ray finned fish.

\section{Acknowledgments}

We thank Manfred Schartl, University of Würzburg, for providing specimens of $X$. montezumae and X. milleri, and Ruth Garcia-Chico for experimental assistance. This work was supported by the University of Konstanz, by a guest professorship to M. M.-B. by the University of Konstanz, and by grants from the Deutsche Forschungsgemeinschaft to A. M.

\section{REFERENCES}

Akimenko, M. A., Johnson, S. L., Westerfield, M., and Ekker, M. 1995. Differential induction of four $m s x$ homeobox genes during fin development and regeneration in zebrafish. Development 121: 347-357.

Andersson, M. 1982. Female choice selects for extreme tail length in a widowbird. Science 299: 818-820.

Angus, A. R., McNatt, H. B., Howell, W. M., and Peoples, S. D. 2001. Gonopodium development in normal male and 11-ketotestosteronetreated mosquitofish (Gambusia affinis): a quantitative study using computer image analysis. Gen. Compar. Endocrinol. 123: 222-234.

Aparicio, S., Chapman, J., Stupka, E., Putnam, N., Chia, J. M., Dehal, P., et al. 2002. Whole-genome shotgun assembly and analysis of the genome of Fugu rubripes. Science 297: 1301-1310.

Basolo, A. L. 1990a. Female preference for male sword length in the green swordtail, Xiphophorus helleri (Pisces: Poeciliidae). Anim. Behav. 40: 332-338.

Basolo, A. L. 1990b. Female preference predates the evolution of the sword in swordtail fish. Science 250: 808-810.

Basolo, A. L. 1991. Male swords and female preferences: response. Science 253: $1426-1427$.

Begemann, G., and Ingham, P. W. 2000. Developmental regulation of Tbx5 in zebrafish embryogenesis. Mech. Dev. 90: 299-304.

Bendall, A. J., and Abate-Shen, C. 2000. Roles for Msx and Dlx homeoproteins in vertebrate development. Gene 247: 17-31.

Borday, V., Thaeron, C., Avaron, F., Brulfert, A., Casane, D., Laurenti, P., and Geraudie, J. 2001. evxl transcription in bony fin rays segment boundaries leads to a reiterated pattern during zebrafish fin development and regeneration. Dev. Dynam. 220: 91-98.

Borowsky, R. L., McClelland, M., Cheng, R., and Welsh, J. 1995. Arbitrarily primed DNA fingerprinting for phylogenetic reconstruction in vertebrates: the Xiphophorus model. Mol. Biol. Evol. 12: 1022-1032.

Darwin, C. 1871. The Descent of Man and Selection in Relation to Sex. John Murray, London.

Dzwillo, M. 1962. Einfluss von Methyltestosteron auf die Aktivierung sekundärer Geschlechtsmerkmale über den arttypischen Zustand hinaus (Untersuchungen an xiphophorinen Zahnkarpfen). Verh. Deutsch. Zool. Gesellsch. Wien 56: 151-159.

Ekker, M., Akimenko, M. A., Allende, M. L., Smith, R., Drouin, G., Langille, R. M., Weinberg, E. S., and Westerfield, M. 1997. Relationships among $m s x$ gene structure and function in zebrafish and other vertebrates. Mol. Biol. Evol. 14: 1008-1022.

Fisher, R. A. 1930. The Genetical Theory of Natural Selection. Oxford University Press, Oxford.

Gordon, M., Cohen, H., and Nigrelli, R. F. 1943. A hormone-produced taxonomic character in Platypoecilius maculatus diagnostic of wild P. xiphidium. Am. Nat. 77: 569-572.

Grobstein, C. 1942. Endocrine and developmental studies of gonopod differentiation in certain poeciliid fishes. J. Exp. Zool. 89: 305-328.

Hopper, A. F. 1949. Development and regeneration of the anal fin of normal and castrate males and females of Lebistes reticulatus. J. Exp. Zool. 110: 299-320.

Hu, G., Lee, H., Price, S. M., Shen, M. S., and Abate-Shen, C. 2001. Msx homeobox genes inhibit differentiation through upregulation of cyclin D1. Development 129: 2373-2384. 
Johnson, S. L., and Bennet, P. 1999. Growth control in the ontogenetic and regenerating zebrafish fin. Methods Cell Biol. 59: 301-311.

Laforest, L., Brown, C. W., Poleo, G., Geraudie, J., Tada, M., Ekker, M., and Akimenko, M. A. 1998. Involvement of sonic hedgehog, patchedl and bmp2 genes in patterning of the zebrafish dermal fin rays. Development 125: 4175-4184.

Marcus, J. M., and McCune, A. R. 1999. Ontogeny and phylogeny in the northern swordtail clade of Xiphophorus. System. Biol. 48: 491-522.

Meyer, A. 1997. The evolution of sexually selected traits in male swordtail fishes (Xiphophorus: Poeciliidae). Heredity 79: 329-337.

Meyer, A., and Lydeard, C. 1993. The evolution of copulatory organs, internal fertilization, placentae and viviparity in killifishes (Cyprinodontiformes) inferred from a DNA phylogeny of the tyrosinase kinase gene $X$-src. Proc. R. Soc. Lond. B 254: 153-162.

Meyer, A., Morrissey, J. M., and Schartl, M. 1994. Recurrent origin of a sexually selected trait in Xiphophorus fishes inferred from a molecular phylogeny. Nature 368: 539-542.

Murciano, C., Fernández, T., Durán, I., Maseda, D., Ruiz, J., Becerra, J., et al. 2003. Ray-interray interactions during fin regeneration of Danio rerio. Dev. Biol. 252: 214-224.

Nechiporuk, A., and Keating, M. T. 2002. A proliferation gradient between proximal and $m s x b$-expressing distal blastema directs zebrafish fin regeneration. Development 129: 2607-2617.

Odelberg, S. J., Kollhof, A., and Keating, M. T. 2000. Dedifferentiation of mammalian myotubes induced by msxl. Cell 103: 1099-1109.
Poss, K. D., Shen, J., Nechiporuk, A., McMahon, G., Thisse, B., Thisse, C., and Keating, M. T. 2000. Roles for FGF signaling during zebrafish fin regeneration. Dev. Biol. 222: 347-358.

Rauchenberger, M., Kallman, K. D., and Morizot, D. C. 1990. Monophyly and geography of the Rio Panuco Basin swordtails (genus Xiphophorus) with descriptions of four new species. Am. Mus. Novit. 2975: 1-41.

Rosen, D. E. 1979. Fishes from the uplands and intermontane basins of Guatemala: revisionary studies and comparative geography. Bull. Am. Mus. Hist. 162: 268-375.

Santamaria, J. A., Mari-Beffa, M., Santos-Ruiz, L., and Becerra, J. 1996. Incorporation of bromodeoxyuridine in regenerating fin tissue of the goldfish Carassius auratus. J. Exp. Zool. 275: 300-307.

Tawk, M., Tuil, D., Torrente, Y., Vriz, S., and Paulin, D. 2002. Highefficiency gene transfer into adult fish: a new tool to study fin regeneration. Genesis 32: 27-31.

Van de Peer, Y., and De Wachter, R. 1993. TREECON: a software package for the construction and drawing of evolutionary trees. Comput. Appl. Biosci. 9: 177-182.

Wang, Y., and Sassoon, D. 1995. Ectoderm-mesenchyme and mesenchymemesenchyme interactions regulate Msx-1 expression and cellular differentiation in the murine limb bud. Dev. Biol. 168: 374-382.

Wiens, J. J., and Morris, M. R. 1996. Character definitions, sexual selection, and the evolution of swordtails. Am. Nat. 147: 866-869.

Zander, C. D., and Dzwillo, M. 1969. Untersuchungen zur Entwicklung und Vererbung des Caudalfortsatzes der Xiphophorus-Arten (Pisces). Zeitschr. Wissensch. Zool. 178: 275-315. 